\title{
FLUPOL \\ Modèle de prévision des débits et des flux polluants en réseaux d'assainissement par temps de pluie Calage et validation
}

\author{
G. Bujon * et L. Herremans **
}

\section{Introduction}

Le ruissellement pluvial urbain est considéré depuis de nombreuses années comme une des sources de dégradation importante des milieux récepteurs, notamment dans les zones les plus fortement urbanisées.

Ainsi n'est-il pas rare d'observer dans les cours d'eau, après des épisodes pluvieux de type orageux, des baisses notables de la teneur en oxygène dissous, teneur pouvant atteindre la limite critique entraînant des mortalités piscicoles.

Les effets de la pollution engendrée par ces eaux sont particulièrement aigus dans les zones à fort niveau d'imperméabilisation, dans celles dont l'assainissement est de type unitaire ou dans celles ayant un milieu récepteur extrêmement sensible. Ils peuvent compromettre les efforts réalisés dans la collecte et le traitement des eaux usées.

Cependant, malgré la prise de conscience, aussi bien des techniciens que des décideurs, de l'importance de ce problème, peu d'actions sont entreprises du fait notamment de la difficulté de quantification de ces phénomènes.

Aussi, avant de projeter des solutions techniques de traitement de ces eaux, il paraît nécessaire de déterminer avec une précision acceptable les débits et les flux polluants. Il semble opportun par ailleurs de tenter d'établir les relations de cause à effet entre pollution rejetée et désordres constatés.
L'une des voies possibles consiste à mesurer concomitamment et systématiquement les flux de pollution amenés par les différents rejets et les concentrations résultantes dans le milieu récepteur.

Cependant cette méthode soulève des difficultés pour des rejets permanents en nombre élevé. Elle devient vite impraticable en période de pluie, à cause du caractère temporaire et éminemment transitoire du phénomène à mesurer.

Une autre voie consiste à mettre au point des outils de modélisation mathématique permettant de quantifier les différents phénomènes mis en jeu. Ce type d'outils nécessite bien évidemment un certain nombre de mesures préalables afin de caler et valider les algorithmes retenus dans le modèle et de permettre son utilisation à d'autres sites.

C'est le cas du modèle FLUPOL, modèle qui décrit les rejets urbains par temps de pluie en liaison avec les caractéristiques de la pluie, des bassins de collecte et du réseau de collecte.

Après un bref rappel descriptif du modèle FLUPOL, son calage et sa validation réalisés à l'aide de mesures effectuées sur différents bassins versants français sont évoqués ciaprès.

(*) Compagnie générale des eaux, détaché à l'agence financière de bassin Seine-Normandie.

$\left({ }^{* *}\right)$ Agence financière de bassin Seine-Normandie.

FLUPOL: A model to forecast flow rates and flows of pollutants in water decontamination networks in time of rain Measuring and validation

The model FLUPOL describes urban pollution in times of rain linking up with the characteristics of rain, collecting basins and the collecting network. It enables simulation, upstream from an urban catchement area decontaminated by one or more networks, of the hydrograms and pollution flows engendered by any rain occurence and characterized by a certain number of variables and takes into account a succession of phenomena from accumulation of waste deposits on the ground to a possible suspension of sediments in the channels. After a short description of the model, its measurement and validation are carried out with the help of different measurements carried out in various French catchement areas. 


\begin{tabular}{|c|c|c|}
\hline \multicolumn{3}{|c|}{ Liste des symboles } \\
\hline AR & ha & Surface du bassin versant homogène \\
\hline $\mathrm{C}_{\mathrm{MAX}}$ & $\mathrm{kg} / \mathrm{m}^{3}$ & Concentration d'équilibre en cas de sédimentation \\
\hline $\mathrm{C}_{\mathrm{MIN}}$ & $\mathrm{kg} / \mathrm{m}^{3}$ & Concentration d'équilibre en cas d'érosion \\
\hline $\mathrm{CHc}$ & $\mathrm{kg}$ & Charge totale calculée \\
\hline $\mathrm{CHm}$ & $\mathrm{kg}$ & Charge totale mesurée \\
\hline Cmac & $\mathrm{mg} / \mathrm{l}$ & Concentration maximum calculée \\
\hline Cmam & $\mathrm{mg} / \mathrm{l}$ & Concentration maximum mesurée \\
\hline $\mathrm{Cmc}$ & $\mathrm{mg} / \mathrm{l}$ & Concentration moyenne calculée sur l'événement \\
\hline $\mathrm{Cmm}$ & $\mathrm{mg} / \mathrm{l}$ & Concentration moyenne mesurée sur l'événement \\
\hline Fmac & $\mathrm{kg} / \mathrm{s}$ & Flux maximum calculé \\
\hline Fmam & $\mathrm{kg} / \mathrm{s}$ & Flux maximum mesuré \\
\hline HPE & $\mathrm{m}$ & Hauteur totale de pluie dans la formule de Desbordes \\
\hline HTP & $\mathrm{mm}$ & Hauteur totale de pluie dans les tableaux 1 à 6 \\
\hline $\mathrm{I}_{\text {MAXS }}$ & $\mathrm{mm} / \mathrm{h}$ & Intensité maximum sur 5 minutes \\
\hline IMPR & - & Coefficient de ruissellement \\
\hline $\mathrm{K}_{\mathrm{DESB}}$ & - & Coefficient multiplicateur de la formule de Desbordes \\
\hline KP & - & $\begin{array}{l}\text { Fraction en poids d'un polluant donné dans les matières } \\
\text { en suspension provenant du sol }\end{array}$ \\
\hline LNG & $\mathrm{m}$ & Longueur maximum d'un sous-bassin homogène \\
\hline MD & $\mathrm{kg} / \mathrm{ha}$ & Masse résiduelle à la fin de la pluie précédente \\
\hline NJ & jour & Nombre de jours de temps sec \\
\hline PNT & $\%$ & Pente moyenne du terrain \\
\hline PS & $\mathrm{kg} / \mathrm{ha} \cdot \mathrm{j}$ & $\begin{array}{l}\text { Masse de poussières et salissures se déposant au sol dans } \\
\text { l'unité de temps et d'espace }\end{array}$ \\
\hline Qmac & $\mathrm{m}^{3} / \mathrm{s}$ & Débit maximum calculé \\
\hline Qmam & $\mathrm{m}^{3} / \mathrm{s}$ & Débit maximum mesuré \\
\hline $\mathrm{T} 3 \mathrm{M}$ & $\mathrm{mn}$ & Durée totale de la pluie dans les tableaux 1 à 6 \\
\hline $\mathrm{T} 3$ & s & Durée totale de la pluie dans la formule de Desbordes \\
\hline Vcal & $\mathrm{m}^{3}$ & Volume total calculé \\
\hline Vmes & $\mathrm{m}^{3}$ & Volume total mesuré \\
\hline Vmesr & $\mathrm{m}^{3}$ & Volume mesuré ruisselé \\
\hline Vplu & $\mathrm{m}^{3}$ & Volume total précipité \\
\hline Vts & $\mathrm{m}^{3}$ & Volume de temps sec \\
\hline$\cap_{1}, \cap_{2}$ & - & Fractions d'énergie totale consommée par l'écoulement \\
\hline
\end{tabular}




\section{Le modèle FLUPOL}

Le modèle FLUPOL permet de simuler, à l'aval d'un bassin versant urbain assaini par un réseau unitaire ou séparatif, les hydrogrammes et les flux de pollution engendrés par un événement pluvieux quelconque (1) (2) et caractérisés par un certain nombre de variables d'état.

Il tient compte d'une succession de phénomènes allant de l'accumulation des dépôts sur le sol à la remise en suspension éventuelle des sédiments dans les conduites.

Les principaux phénomènes simulés sont les suivants :

- calcul de l'évolution temporelle des dépôts entre deux pluies consécutives d'une part sur le sol en fonction des caractéristiques d'occupation du sol et, d'autre part, dans les conduites d'assainissement en fonction des caractéristiques de celles-là et du flot de temps sec éventuel ;

- calcul des apports en débit et flux de pollution de sous-bassins élémentaires à partir des caractéristiques d'une pluie donnée et de celles des sous-bassins (calcul suivant le modèle dit de réservoir linéaire pour les débits et d'un double réservoir linéaire pour les flux polluants);

- à chaque næud du réseau, bilan des débits et des flux de pollution entrants et sortants en fonction des caractéristiques de chacun de ces nœuds (affluence, déversoir, dérivation, confluence, station de pompage, etc...) ;

- propagation dans chaque tronçon de conduite des débits et des flux de pollution en tenant compte du dépôt ou de la remise en suspension éventuel des sédiments.

Le modèle permet actuellement de simuler, outre le débit, l'évolution des quatre variables d'état suivantes : les matières en suspension, la DBO5, la DCO et l'azote Kjeldahl.

Le lecteur trouvera dans l'article de G. Bujon (2) une explication détaillée de FLUPOL, notamment des différents phénomènes et des algorithmes qui s'y rattachent, en sachant par ailleurs que certaines améliorations sont intervenues depuis sa parution.

Ces améliorations portent, en particulier, sur un nouveau mode de simulation des phénomènes liés aux déversoirs d'orage et sur la possibilité de prendre en compte des stations de pompage.

\section{Les bassins versants étudiés}

Le calage et les validations du modèle FLUPOL sont réalisés à l'aide de mesures (3) (4) (5) (6) effectuées sur quatre bassins versants d'occupation de sols et de dimensions très différentes.

Ces bassins, situés dans la région Ile de France, sont les suivants :

- Bassin du collecteur des Coteaux (Paris);

- Bassin de Maurepas (département des Yvelines);

- Bassin des Ulis Nord (département de l'Essonne);

- Bassin du collecteur Fresnes-Choisy (départements des Hauts-de-Seine et du Val de Marne).
Chacun de ces bassins a fait l'objet de mesures hydrauliques, chimiques et biochimiques : en 1979 pour le collecteur des Coteaux, en 1980 et 1982 pour Maurepas, en 1982 pour les Ulis et en 1986 pour le collecteur Fresnes-Choisy.

\section{Le Bassin du collecteur des Coteaux}

Ce bassin versant est situé dans le $12^{\mathrm{e}}$ arrondissement de Paris (75). Lors des mesures, la configuration du réseau de ce secteur avait été modifiée afin que le bassin versant étudié soit parfaitement délimité (suppression de tout maillage), la superficie résultante étant alors de 110 hectares. L'occupation du sol est de type résidentiel dense (250 habitants/hectare) et constituée essentiellement d'immeubles d'habitation à couverture classique en ardoises engendrant un coefficient de ruissellement très élevé $(0,85)$.

Le réseau est de type unitaire et draine un flot de temps sec de l'ordre de $280 \mathrm{l} / \mathrm{s}$.

La longueur totale de réseau prise en compte dans les simulations est de 1600 mètres.

Un ensablement important est constaté lors des mesures. Son origine est attribuée à la faible pente des ouvrages de la partie aval (de l'ordre de 0,0005 ). Cet ensablement peut avoir des conséquences significatives sur les simulations.

Enfin, par temps de pluie, le réseau peut être délesté vers la Seine par l'intermédiaire d'un grand déversoir d'orage nommé "déversoir Proudhon" comportant plusieurs baies de déversement d'une longueur totale d'environ 18 mètres.

\section{Le bassin de Maurepas}

Ce bassin versant est situé dans le département des Yvelines (78) à l'Ouest de Paris et fait partie de la ville nouvelle de Saint-Quentin-en-Yvelines. Il couvre une superficie de 26,7 hectares et est composé d'un habitat résidentiel discontinu (100 habitants/hectare) formé pour $80 \%$ d'une zone pavillonnaire à couverture classique et pour $20 \%$ d'une zone de bâtiments collectifs à usage d'habitation dont la couverture est de type toiture-terrasse. La prédominance des bâtiments à couverture classique en tuiles a pour conséquence un coefficient de ruissellement assez élevé $(0,70)$.

Situé en zone nouvelle, le réseau d'assainissement était, lors des mesures, strictement séparatif (seul un petit débit d'eau de drainage avait été détecté).

La longueur total de réseau prise en compte dans les simulations est de 800 mètres, les canalisations ayant un diamètre variant entre $800 \mathrm{~mm}$ et $1160 \mathrm{~mm}$.

\section{Le bassin des Ulis Nord}

Ce bassin versant est situé dans le département de l'Essonne (91) au Sud de Paris. Il couvre une superficie de 43,1 hectares et est composé d'un habitat collectif assez dense ( 350 habitants/hectare). La couverture des bâtiments est du type toiture-terrasse, type qui explique le coefficient de ruissellement assez faible $(0,42)$. 
Bien que situé en zone nouvelle, le réseau d'assainissement n'est pas strictement séparatif, le réseau pluvial transitant un flot de temps sec d'environ $31 / \mathrm{s}$. Ce flot, bien que faible, engendre cependant des dépôts, notamment à l'extrémité du réseau à très faible pente.

La longueur totale de réseau prise en compte dans les simulations est de 2750 mètres, les canalisations ayant un diamètre variant entre $600 \mathrm{~mm}$ et $1800 \mathrm{~mm}$.

\section{Le bassin du collecteur Fresnes-Choisy}

Le bassin du collecteur Fresnes-Choisy (2) (7) (8) est situé dans la banlieue Sud de Paris et concerne plusieurs départements. Cependant, pour des commodités de simulation, il est possible de limiter la zone aux seuls départements des Hauts-de-Seine et du Val-de-Marne, la zone ainsi couverte représentant près de 2500 hectares. L'occupation du sol est très hétérogène : habitat pavillonnaire discontinu, habitat collectif dense, autoroutes urbaines, zones industrielles et zones d'activités tertiaires. Cependant, compte tenu de la densité moyenne d'habitants par hectare (100 habitants/hectare), on considère que l'ensemble du bassin est de type résidentiel discontinu.

Des études antérieures (7) (8) montrent que le coefficient de ruissellement de chacun des sous-bassins peut être déduit du coefficient d'imperméabilisation par une formule du type :

$$
\text { Cruissellement }=\mathrm{a} * \text { Cimperméabilisation }+\mathrm{b}
$$

a et b étant deux coefficients calés à partir de mesures pluies-débits.

Le réseau de type pluvial comporte cependant un flot de temps sec de l'ordre de $75 \mathrm{l} / \mathrm{s}$.

La longueur totale du réseau prise en compte est d'environ $23 \mathrm{~km}$, le diamètre des ouvrages variant de $600 \mathrm{~mm}$ à $4000 \mathrm{~mm}$.

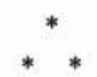

En résumé, les caractéristiques principales des bassins sont les suivantes :

\begin{tabular}{|c|c|c|c|c|}
\hline & $\begin{array}{c}\text { Les } \\
\text { Coteaux }\end{array}$ & Maurepas & $\begin{array}{c}\text { Les Ulis } \\
\text { Nord }\end{array}$ & $\begin{array}{l}\text { Fresnes- } \\
\text { Choisy }\end{array}$ \\
\hline Surface ha & 110,00 & 26,70 & 43,10 & 2486 \\
\hline Nombre hab./ha & 250 & 100 & 350 & 100 \\
\hline IMPR moyen & 0,85 & 0,70 & 0,42 & 0,42 \\
\hline $\begin{array}{l}\text { Type de réseau } \\
\text { Flot temps sec }\end{array}$ & unitaire & séparatif & séparatif & séparatif \\
\hline
\end{tabular}

L'examen comparatif des 4 bassins versants étudiés montre que le modèle est confronté à des situations présentant :

- une occupation des sols très contrastée, le coefficient d'imperméabilisation moyen variant de 0,42 à 0,85 et le nombre d'habitants à l'hectare variant, quant à lui, de 100 à 350 ;
- des tailles de bassins allant de faible à très importante ; - des réseaux aussi bien unitaires que séparatifs ayant ou non des débits de temps sec.

\section{Le calage}

L'objectif visé lors de l'établissement du présent modèle de simulation est la création d'un outil applicable au plus grand nombre de cas, sans qu'il soit nécessaire de retoucher à certains paramètres internes indépendants des caractéristiques spécifiques du cas étudié.

Aussi est-il nécessaire, avant d'utiliser un modèle au niveau opérationnel, de passer par un stade préalable de calage, stade permettant de régler certains paramètres en vue d'améliorer l'ajustement du modèle à des données expérimentales.

Ce réglage est une tâche qui mérite d'être effectuée sur le plus grand nombre de cas possibles; elle est délicate, car il est difficile de trouver un jeu de paramètres satisfaisant à tous les cas considérés.

Dans le cas du modèle FLUPOL, le calage du modèle est réalisé à partir de deux jeux de données expérimentales provenant de certains des bassins versants précédemment cités, à savoir :

- pour le bassin de Maurepas, l'événement du 19 septembre 1980 ;

- pour le bassin des Ulis-Nord, l'événement du 21 mai 1982.

L'ajustement du paramètre KP est effectué à l'aide d'autres mesures que celles de ces deux campagnes pour des raisons explicitées ci-après.

Ce calage, dont les résultats figurent sur les graphiques I et 2 (voir p. 130 et 131), a porté sur les paramètres PS, $\mathrm{KP}, \mathrm{K}_{\mathrm{DESB}}, \cap_{1}$ et $\cap_{2}$.

\subsection{La quantité spécifique de dépôts sur le sol PS en fonc- tion de l'occupation du sol}

Dans un premier temps, les simulations (2) sont réalisées à partir de valeurs provenant de la bibliographie américaine.

Il y a quatre types de zones : résidentiel dense, résidentiel discontinu, commercial et industriel. Les valeurs de PS sont fournies pour chacun d'eux en masse de dépôts par jour et par unité de longueur de trottoir.

Transposées en masse de dépôts par jour et par hectare, les valeurs initialement prises en compte sont les suivantes :

résidentiel dense

résidentiel discontinu

$10 \mathrm{~kg} / \mathrm{j} / \mathrm{ha}$

commercial

$5 \mathrm{~kg} / \mathrm{j} / \mathrm{ha}$

industriel

$15 \mathrm{~kg} / \mathrm{j} / \mathrm{ha}$

$35 \mathrm{~kg} / \mathrm{j} / \mathrm{ha}$

Compte tenu des données expérimentales disponibles, le calage ne porte que sur les deux premiers types de zone et conduit à adopter pour ceux-là les valeurs sensiblement différentes ci-après : 
résidentiel dense résidentiel discontinu

$25 \mathrm{~kg} / \mathrm{j} / \mathrm{ha}$

$6 \mathrm{~kg} / \mathrm{j} / \mathrm{ha}$

Pour les zones de type commercial ou industriel, les valeurs de la bibliographie américaine ont été conservées. Des campagnes de mesures spécifiques devront être menées pour affiner ces valeurs.

\subsection{La fraction en poids KP d'un polluant donné dans les matières en suspension provenant du sol}

Comme précédemment, les simulations initiales sont réalisées à partir de données bibliographiques prenant comme hypothèse que cette fraction est constante quel que soit l'événement simulé. La comparaison des résultats obtenus sur cette base avec les valeurs expérimentales utilisées pour le calage montre l'inexactitude de cette hypothèse et a conduit à retenir un coefficient KP variable en fonction des caractéristiques de la pluie.

La relation adoptée pour l'évaluation de ce coefficient KP fait intervenir l'intensité maximale de la pluie sur 5 min, $\mathrm{I}_{\text {MAXs } 5}$, atteinte au cours de l'événement donnant lieu à simulation.

La relation reliant $\mathrm{KP}$ et $\mathrm{I}_{\mathrm{MAx} \text { s }}$ est de la forme :

$$
\mathrm{KP}=\mathrm{a} *\left(\mathrm{I}_{\mathrm{MAXs}}-\mathrm{c}\right)^{-\mathrm{b}}+\mathrm{d} .
$$

Cette relation permet de transcrire l'idée selon laquelle les pluies les plus intenses entraînent plus facilement des particules de forte densité à prédominance minérale et moins chargées en matières organiques carbonées ou azotées.

L'ajustement a été réalisé à partir des mesures du bassin de Maurepas pour lequel on disposait d'une année complète de mesures (pluie et charges totales en MES, DBO5 et DCO), charges non influencées par des remises en suspension éventuelles, ce bassin étant doté d'un réseau strictement séparatif.

Il aboutit aux formules suivantes :

$\begin{array}{ll}\text { DBO5 } & : \mathrm{KP}=0,28 * \mathrm{I}_{\mathrm{MAX5}}{ }^{-0,572} \\ \mathrm{DCO} & \mathrm{KP}=1,47 * \mathrm{I}_{\mathrm{MAX5}}{ }^{-0,419}\end{array}$

Ces formules sont considérées comme applicables aux zones de type résidentiel au sens large.

Dans le cas des zones de types commercial et industriel, l'absence de mesure ne permet pas, pour l'instant, de déterminer des lois correspondantes.

L'absence de mesures des paramètres azotés sur le bassin de Maurepas empêche également la réalisation d'un ajustement similaire pour l'azote Kjeldahl.

Aussi, dans le cas du bassin du collecteur FresnesChoisy, on est amené à adopter une formulation légèrement différente (le bassin comportant des zones relativement variées) pour la $\mathrm{DBO} 5$ et la $\mathrm{DCO}$ et à réaliser un ajustement pour le NTK.

$\begin{array}{ll}\text { DBO5 } & \text { KP }=0,17 *\left(\mathrm{I}_{\text {MAX } 5}-0,9\right)^{-0,700}+0,05 \\ \text { DCO } & \text { KP }=0,93 *\left(\mathrm{I}_{\text {MAX } 5}-0,5\right)^{-0,700}+0,44 \\ \text { NTK } & : K P=0,07 *\left(\mathrm{I}_{\text {MAX } 5}-0,8\right)^{-0,600}+0,001 .\end{array}$

Les ajustements concernant la DBO5 et la DCO sont peu différents de ceux déterminés pour les zones de type résidentiel. Ils permettent cependant de prendre globale- ment en compte l'ensemble des zones existant sur le bassin du Fresnes-Choisy.

Les graphiques ci-dessous donnent les courbes d'ajustement pour les 3 variables d'état DBO5, DCO et NTK.


\subsection{La valeur du coefficient multiplicateur de la formule de Desbordes}

M. Desbordes (1) propose dans sa thèse une formule comportant un coefficient $\mathrm{K}$ de proportionnalité entre le volume d'eau stocké à un instant donné sur une zone définie et le débit qui en sort au même instant. 
Cette formule est la suivante :

$$
\begin{gathered}
\mathrm{K}=\mathrm{KDESB} * \mathrm{AR}^{0,18} * \mathrm{PNT}^{-0,36} *\left(1+\mathrm{IMPR}^{-1,9} *\right. \\
\mathrm{T} 3^{0,21} * \mathrm{LNG}^{0,15} * \mathrm{HPE}^{-0,07} .
\end{gathered}
$$

Compte tenu des unités adoptées dans FLUPOL pour les différents termes de cette formule, le coefficient $\mathrm{K}_{\mathrm{DESB}}$ correspondant a pour valeur 79,35.

D'une façon générale, cette valeur fournit de bons résultats mais minimise légèrement les débits de pointe. Aussi on a choisi d'adopter une valeur un peu plus faible égale à 50 .

A titre d'illustration, dans le cas particulier de la pluie du 19 septembre 1980 à Maurepas, le graphique ci-dessous montre l'incidence de la modification de ce paramètre sur l'hydrogramme à l'exutoire du bassin.

MAUREPAS*PLUIE DU 19 SEPTEMBRE 1980

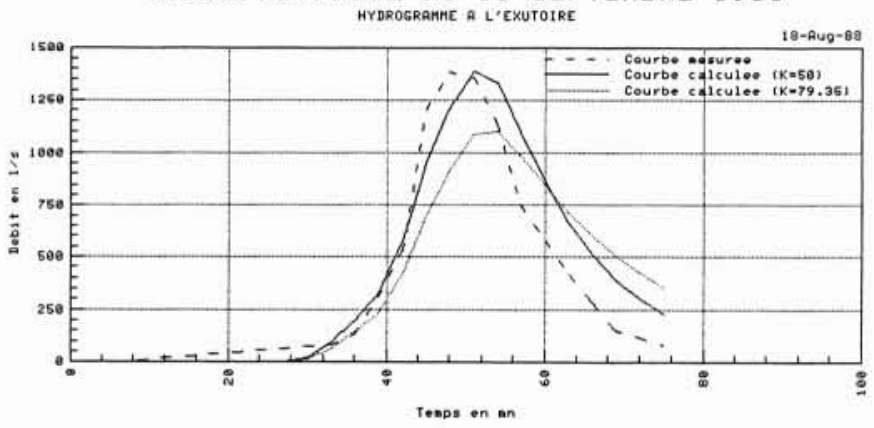

\subsection{Calage des coefficients $\cap_{1}$ et $\cap_{2}$ déterminant les condi- tions de dépôts ou remises en suspension}

Rappelons brièvement que $\bigcap_{1}$ et $\bigcap_{2}$ sont des coefficients de proportionnalité qui lient la concentration de MES dans l'écoulement à :

- la vitesse de chute des particules sédimentables;

- la vitesse d'écoulement de l'eau ;

- la perte de charge.

Cette notion de proportionnalité revient à considérer qu'une fraction constante de l'énergie sert soit à maintenir des particules sédimentables en suspension $\left(\bigcap_{1}\right)$, soit à mettre en suspension et à les y maintenir des particules précédemment sédimentées $\left(\cap_{2}\right)$.

Le calage effectué conduit à :

$$
\cap_{1}=0,022 \quad \cap_{2}=0,018 .
$$

\section{La validation}

La validation du modèle consiste à comparer le modèle, préalablement calé à l'aide de données expérimentales particulières, à d'autres données expérimentales n'ayant pas servi au calage. Cette validation est réalisée sur les quatre bassins versants décrits précédemment.
Pour chacun d'eux, on dispose d'un certain nombre de mesures portant sur la pluviométrie, le débit et les flux polluants à l'aval du bassin versant.

Préalablement à chaque essai de validation, il est indispensable de connaître, à la fin de la pluie précédant la pluie à simuler, à la fois le degré de pollution du sol et la quantité de dépôts dans les différents tronçons du réseau. Il est donc nécessaire de procéder à une simulation continue de l'ensemble des pluies précédant celle à simuler et ceci à partir d'un événement antérieur suffisamment intense. Ainsi au début de l'événement choisi, on détermine :

- d'une part, le degré de salissure du sol des différents sous-bassins ;

- d'autre part, l'encrassement éventuel des différents tronçons du réseau.

Un grand nombre de simulations de validation, chacune correspondant à un bassin et une pluie donnés, a été réalisé pour les quatre bassins précités; cinq d'entre elles sont choisies pour être présentées ici.

Il est bon de souligner que ces simulations sont réalisées avec un modèle identique quels que soient le bassin et la pluie étudiés.

Les événements simulés sont les suivants :

Bassin du collecteur Fresnes-Choisy

Pluie du 22 août 1986

Pluie du 15 septembre 1986

Pluie du 20 octobre 1986.

Bassin du collecteur des Coteaux

Pluie du 7 août 1979.

Bassin des Ulis-Nord

Pluie du 28 septembre 1982.

Les résultats figurent dans les graphiques 3 à 7 (p. 132 à 136) sur lesquels sont indiqués pour chaque événement :

- le ou les hyétogrammes utilisés (à l'exception de l'événement du bassin du collecteur des Coteaux pour lequel on reconstitue une pluie de projet à partir de données de postes pluviographiques proches du site de mesures) ;

- les hydrogrammes calculé et mesuré ;

- les courbes de variation des flux de MES, DBO5, DCO et NTK (le NTK ne figurant que pour le Fresnes-Choisy) calculées et mesurées.

Par ailleurs, les tableaux 1 à 6 (p. 137 à 139) donnent les principales caractéristiques résultant de chacune des simulations, soit :

Tableau 1 : Caractéristiques de la pluie et des dépôts sur le sol.

Tableau 2 : Résultats globaux concernant les volumes et les débits calculés et mesurés.

Tableaux 3 à 6 : Résultats globaux concernant les charges et les flux de pollution calculés et mesurés pour chacun des paramètres simulés.

Ces différents résultats font apparaître une concordance relativement bonne entre calculs et mesures quel que soit le paramètre. En particulier, la différence entre la charge totale calculée et celle mesurée est, dans la plupart des cas, inférieure à $20 \%$. 
La concomitance entre pointe de flux et pointe de débit est la règle générale (comme le montre la pluie du 21 mai 1982 sur le bassin des Ulis-Nord). Si on raisonne en terme de flux, cela infirme la notion selon laquelle le premier flot d'orage (le first flush des anglo-saxons) serait le plus chargé en pollution. Les flux les plus importants étant ceux pouvant entraîner les conséquences les plus dommageables pour le milieu récepteur, ce ne sont pas obligatoirement les premiers flots d'orage qui sont les plus nocifs (cette notion de first flush est, par contre, vraie, dans la plupart des cas, en terme de concentration).

Enfin on constate que les algorithmes adoptés pour les phénomènes de dépôt-remise en suspension donnent des résultats satisfaisants sur les bassins sur lesquels des dépôts en réseau sont constatés (Collecteur des Coteaux et Les Ulis-Nord)

\section{Conclusion}

Les résultats présentés font apparaître le modèle FLUPOL sous un jour plutôt favorable, ces résultats étant obtenus sur des bassins versants de caractéristiques très dissemblables.

Il n'est pas impossible, cependant, que la confrontation à des données expérimentales provenant d'autres bassins versants entraîne le changement ou l'ajout de certains algorithmes et prescrive certaines modifications du modèle actuel.

Quoi qu'il en soit, l'utilisation opérationnelle du modèle FLUPOL est actuellement en cours sur l'ensemble du réseau d'assainissement de l'agglomération parisienne. Une partie importante du réseau unitaire parisien est déjà schématisée et a donné lieu à des simulations sur les bases de certaines pluies historiques. Les premiers résultats donnent d'ores et déjà un ordre de grandeur des flux de pollution évacués vers la Seine par l'intermédiaire des principaux déversoirs d'orage parisiens.

\section{Bibliographie}

[1] Desbordes M. - Réflexion sur les méthodes de calcul des réseaux urbains d'assainissement pluvial. Université des sciences et techniques du Languedoc-Montpellier. Thèse de Docteur-Ingénieur, 224 p., février 1974.

[2] Bujon G. - Prévision des débits et des flux polluants transités par les réseaux d'égouts par temps de pluie. Le modèle FLUPOL. La Houille Blanche, pp. 11-23, N 1-1988.

[3] Hemain J.C. - Mesure de la pollution du ruissellement pluvial urbain. Suivi et bilan de la campagne expérimentale. Ministère de l'Urbanisme et du Logement. Service technique de l'urbanisme, 74 p., juillet 1983

[4] Hemain J.C. - Mesure de la pollution du ruissellement pluvial urbain. Exploitation des données. Ministère de l'Urbanisme et du Logement. Service technique de l'urbanisme, 38 p. + annexes, août 1983.

[5] RenaRd D. et al. - Mesures de débit-pollution dans le collecteur des Coteaux et le déversoir Proudhon. LROP, $45 \mathrm{p}$. + annexes, décembre 1980

[6] Grange D. et al. - Mesure de l'impact des eaux pluviales d'un grand bassin versant urbain sur la Seine: cas du bassin du collecteur Fresnes-Choisy. AFBSN-LROP, 45 p. + annexes, juin 1987.

[7] Faure-Soulet A. et al. - Etude de l'émissaire FresnesChoisy. CETE d'Aix-en-provence - DDE du Val-de-Marne, $16 \mathrm{p} .+$ annexes, janvier 1983.

[8] Herremans L. et al. - Simulation du fonctionnement des réseaux pluviaux: deux expériences de calage de modèles. DDE du Val-de-Marne - CETE d'Aix-en-Provence SAFEGE - 8 p., XVII Journées de l'hydraulique de la SHF, Nantes, septembre 1982.

Adresses des auteurs

Monsieur G. Bujon, Agence de bassin Seine-Normandie, 51, rue Salvador-Allende, 92027 Nanterre Cedex, Tél.: (1) 47764424.

Monsieur L. Herremans, Anjou-Recherche, 52, rue d'Anjou, 75008 Paris, Tél.: (1) 42669150.






\section{Graphique 1}

\section{Bassin de Maurepas Pluie du 19 septembre 1980 \\ Phase de validation}
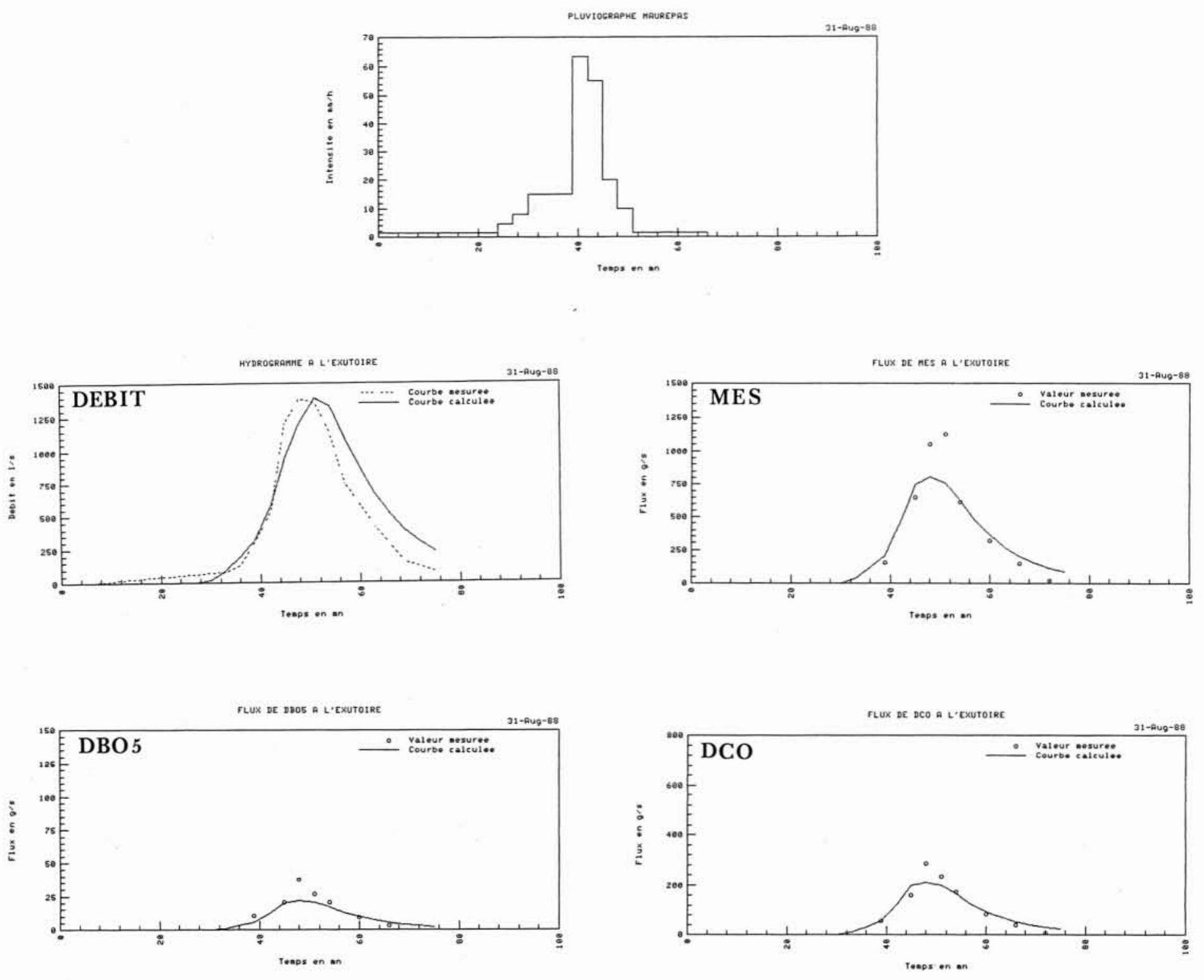

LA HOUILLE BLANCHE/N²-1990 


\section{Graphique 2}

\section{Bassin des Ullis Nord $\quad$ Pluie du 21 mai 1982}

\section{Phase de validation}
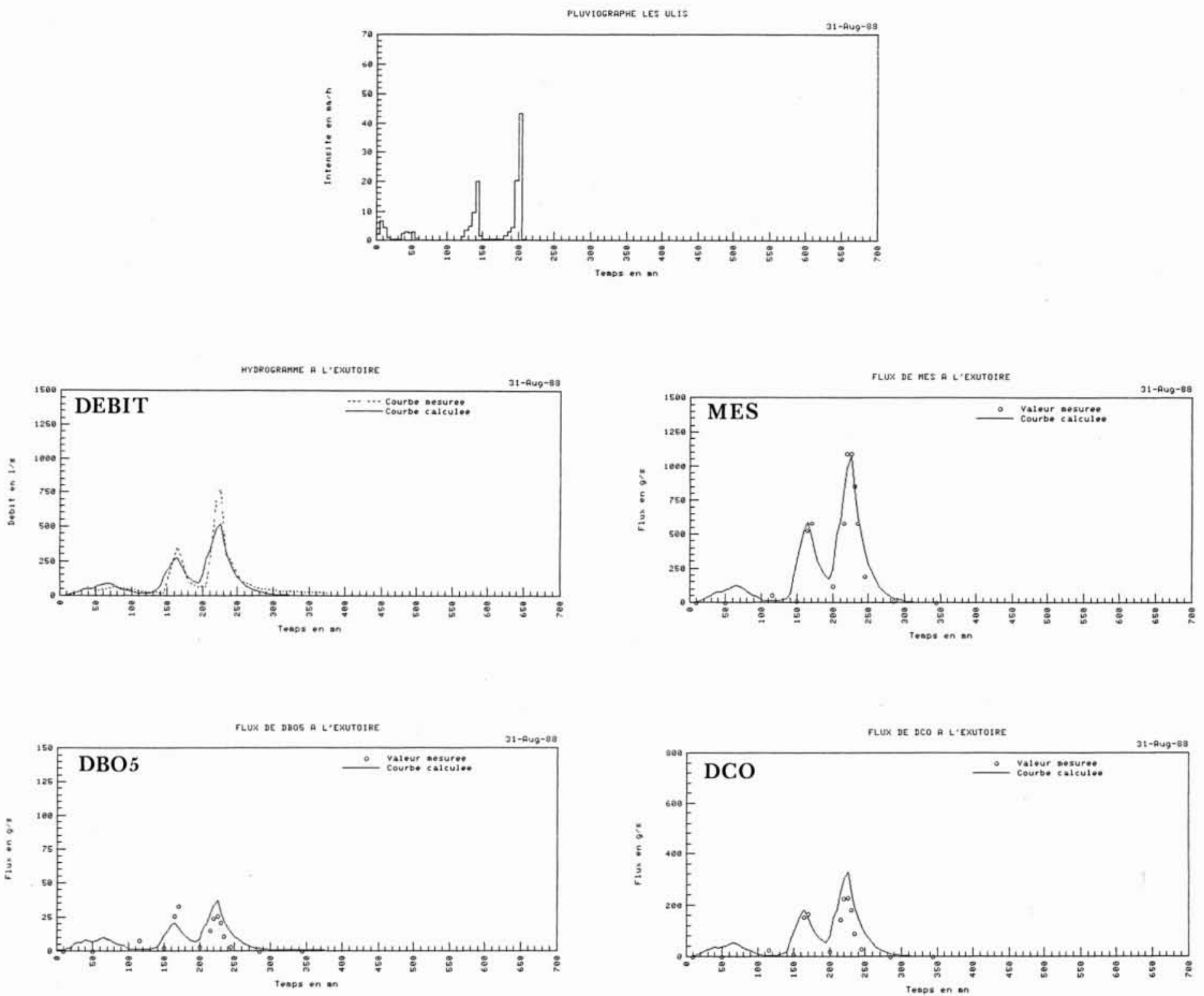

LA HOUILLE BLANCHE/N²-1990 


\section{Graphique 3}

\section{Bassin du collecteur Fresnes-Choisy Pluie du 22 août 1981}

\section{Phase de validation}


LA HOUILLE BLANCHE $/ \mathrm{N}^{\circ} 2-1990$ 


\section{Graphique 4}

\section{Bassin du collecteur Fresnes-Choisy \\ Pluie du 15 septembre 1986}

\section{Phase de validation}
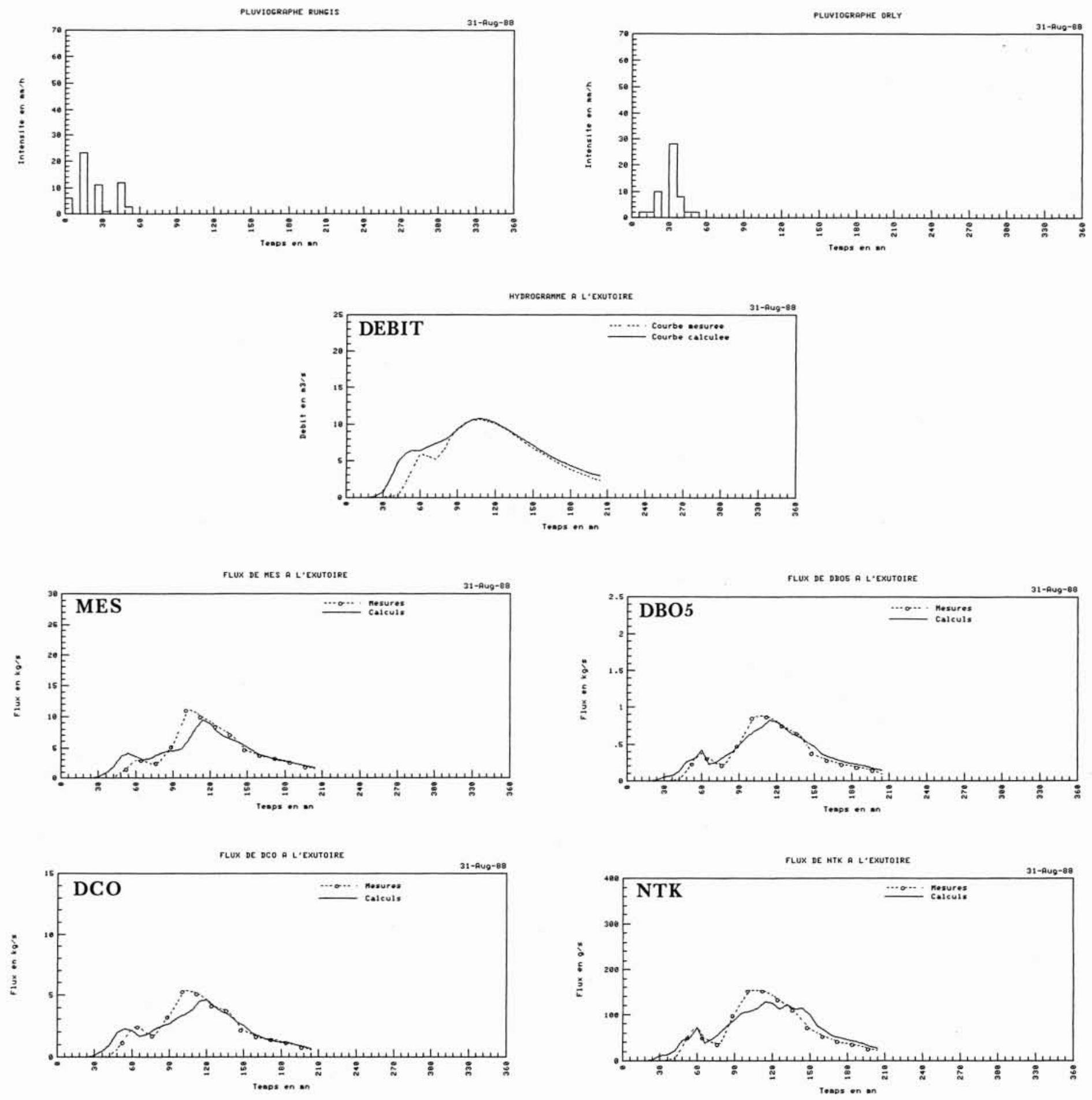

LA HOUILLE BLANCHE/N²-1990 


\section{Graphique 5}

\section{Bassin du collecteur Fresnes-Choisy \\ Pluie du 20 octobre 1986}

\section{Phase de validation}


LA HOUILLE BLANCHE/N²-1990 


\section{Graphique 6}

\section{Bassin du collecteur des Coteaux-Déversoir Proudhon Pluie du 7 août 1979}

\section{Phase de validation}
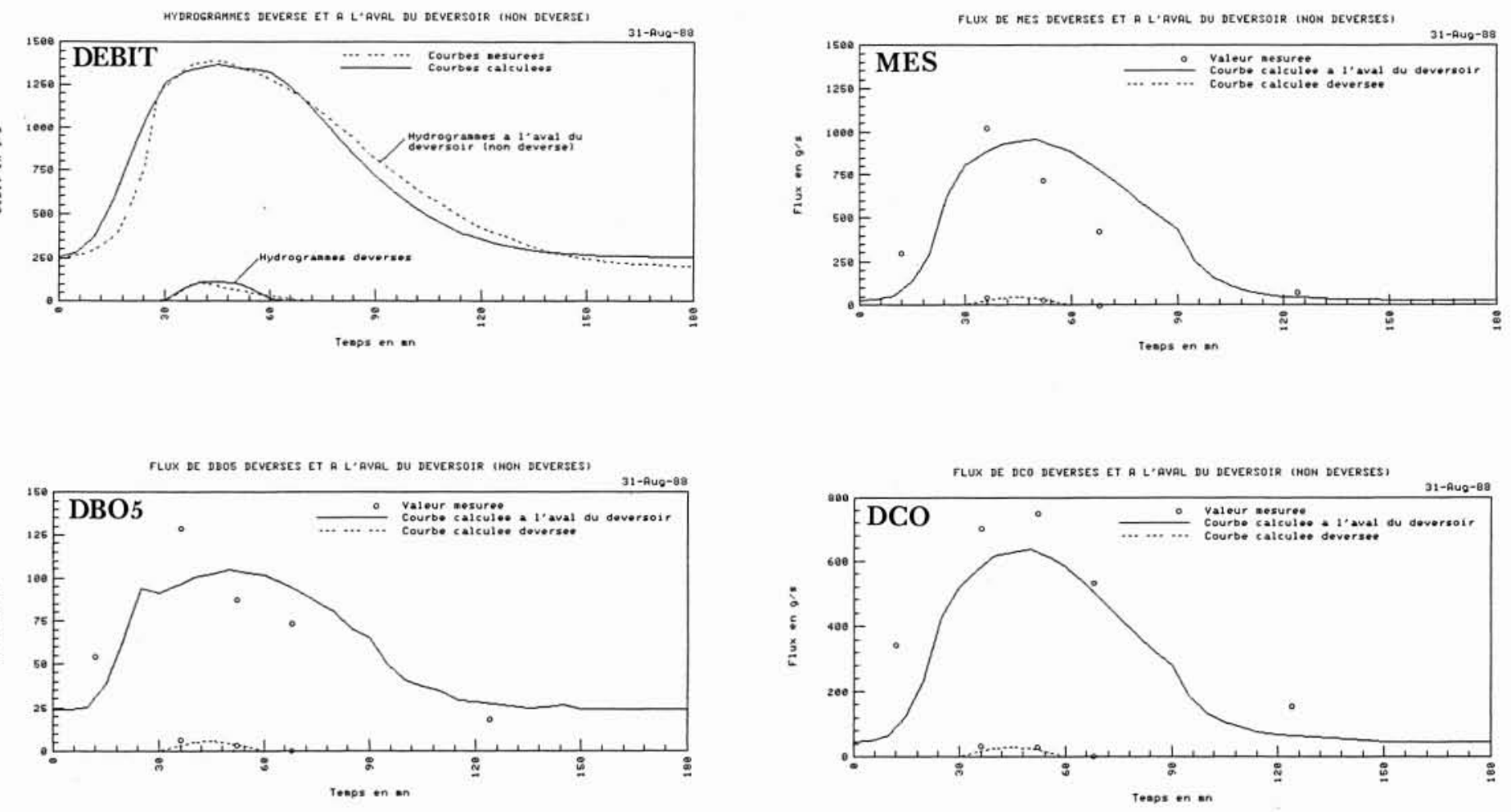

LA HOUILLE BLANCHE/N²-1990 


\section{Graphique 7}

\section{Bassin des Ullis-Nord Pluie du 28 septembre 1982 \\ Phase de validation}


LA HOUILLE BLANCHE/N²-1990 
TABLEAU 1 * : CARACTERISTIQUES DE LA PLUIE ET DES DEPOTS

\begin{tabular}{|c|c|c|c|c|c|c|c|c|}
\hline \multirow{3}{*}{ DATE } & \multicolumn{3}{|c|}{ PRESUES-CHOISY } & \multirow{2}{*}{\multicolumn{2}{|c|}{$\begin{array}{c}\text { LES COTEAUL } \\
0========= \\
07.08 .79\end{array}$}} & \multirow{3}{*}{$\begin{array}{l}\text { MAURBPAS } \\
19.09 .80\end{array}$} & \multicolumn{2}{|c|}{ LRS ULIS } \\
\hline & \multirow{2}{*}{22.08 .80} & \multirow{2}{*}{15.09 .86} & \multirow{2}{*}{20.10 .86} & & & & \multirow{2}{*}{21.05 .82} & \multirow{2}{*}{28.09 .82} \\
\hline & & & & DEVBESE & AVAL DEV & & & \\
\hline aTp & 15,00 & 5,50 & 9,00 & \multicolumn{2}{|c|}{5,30} & 10,40 & 11,90 & 20,00 \\
\hline$I_{\text {XuYs }}$ & 16,00 & 25,50 & 44,00 & \multicolumn{2}{|c|}{7,20} & 59,80 & 43,20 & 56,05 \\
\hline Т3H & 270 & 54 & 78 & \multicolumn{2}{|c|}{80} & 66 & 210 & 500 \\
\hline Vplu & 372900 & 136730 & 238656 & \multicolumn{2}{|c|}{5830} & 2777 & 5129 & 8577 \\
\hline$M D$ & 0,00 & 40,00 & 55,00 & \multicolumn{2}{|c|}{25,00} & 40,00 & 180,00 & 145,00 \\
\hline NJ & 60,00 & 1,90 & 0,15 & \multicolumn{2}{|c|}{0,10} & 8,00 & 2,40 & 1,90 \\
\hline
\end{tabular}

TABLEAU $2 *$ : VOLUMES ET DEBITS




TABLEAU 3 * : MATIERES EN SUSPENSION (M.E.S.T)

\begin{tabular}{|c|c|c|c|c|c|c|c|c|}
\hline \multirow{3}{*}{ DATB } & \multicolumn{3}{|c|}{ FRESHES-CHOISY } & \multirow{2}{*}{\multicolumn{2}{|c|}{$\begin{array}{l}\text { LES COTEAUU } \\
07.08 .79\end{array}$}} & \multirow{3}{*}{$\begin{array}{l}\text { MAURBPAS } \\
19.09 .30\end{array}$} & \multicolumn{2}{|c|}{ LES ULIS } \\
\hline & \multirow{2}{*}{22.08 .86} & \multirow{2}{*}{15.09 .86} & \multirow{2}{*}{20.10 .86} & & & & \multirow{2}{*}{21.05 .82} & \multirow{2}{*}{28.09 .82} \\
\hline & & & & DBVERSZ & AVAL DBV & & & \\
\hline $\begin{array}{l}\mathrm{CHa} \\
\mathrm{CHc} \\
\mathrm{CHc} / \mathrm{CHa}\end{array}$ & $\begin{array}{r}76432 \\
80942 \\
1,14\end{array}$ & $\begin{array}{r}47039 \\
45819 \\
0,97\end{array}$ & $\begin{array}{r}111235 \\
95817 \\
0,86\end{array}$ & $\begin{array}{r}86 \\
50 \\
0,58\end{array}$ & $\begin{array}{l}3711 \\
3687 \\
0,99\end{array}$ & $\begin{array}{r}967 \\
970 \\
1,00\end{array}$ & $\begin{array}{l}3151 \\
3874 \\
1,23\end{array}$ & $\begin{array}{l}3028 \\
3419 \\
1,13\end{array}$ \\
\hline $\begin{array}{l}\text { Paaz } \\
\text { Paac } \\
\text { Paac/Faan }\end{array}$ & $\begin{array}{r}11,080 \\
11,160 \\
0,96\end{array}$ & $\begin{array}{r}11,060 \\
3,480 \\
0,80\end{array}$ & $\begin{array}{r}26,430 \\
19,580 \\
0,74\end{array}$ & $\begin{array}{r}0,052 \\
0,047 \\
0,90\end{array}$ & $\begin{array}{r}1,031 \\
0,957 \\
0,93\end{array}$ & $\begin{array}{r}1,131 \\
0,808 \\
0,71\end{array}$ & $\begin{array}{r}1,097 \\
1,076 \\
0,98\end{array}$ & $\begin{array}{r}1,306 \\
1,236 \\
0,95\end{array}$ \\
\hline $\begin{array}{l}\text { Caan } \\
\text { Canc } \\
\text { Caac/Caan } \\
\text { Cam } \\
\text { Cac } \\
\text { Cac/Can }\end{array}$ & $\begin{array}{r}656 \\
693 \\
1,06 \\
328 \\
366 \\
1,12\end{array}$ & $\begin{array}{r}1056 \\
891 \\
0,84 \\
747 \\
631 \\
0,84\end{array}$ & $\begin{array}{r}1090 \\
911 \\
0,84 \\
799 \\
589 \\
0,36\end{array}$ & $\begin{array}{r}780 \\
429 \\
0,55 \\
541 \\
347 \\
0,64\end{array}$ & $\begin{array}{r}799 \\
707 \\
0,88 \\
508 \\
502 \\
0,99\end{array}$ & $\begin{array}{r}856 \\
802 \\
0,94 \\
635 \\
536 \\
0,84\end{array}$ & $\begin{array}{l}2082 \\
2211 \\
1,06 \\
1354 \\
1824 \\
1,35\end{array}$ & $\begin{array}{r}2900 \\
2732 \\
0,94 \\
779 \\
883 \\
1,13\end{array}$ \\
\hline
\end{tabular}

TABLEAU 4 * : DEMANDE BIOCHIMIQUE EN OXYGENE (DBO5)

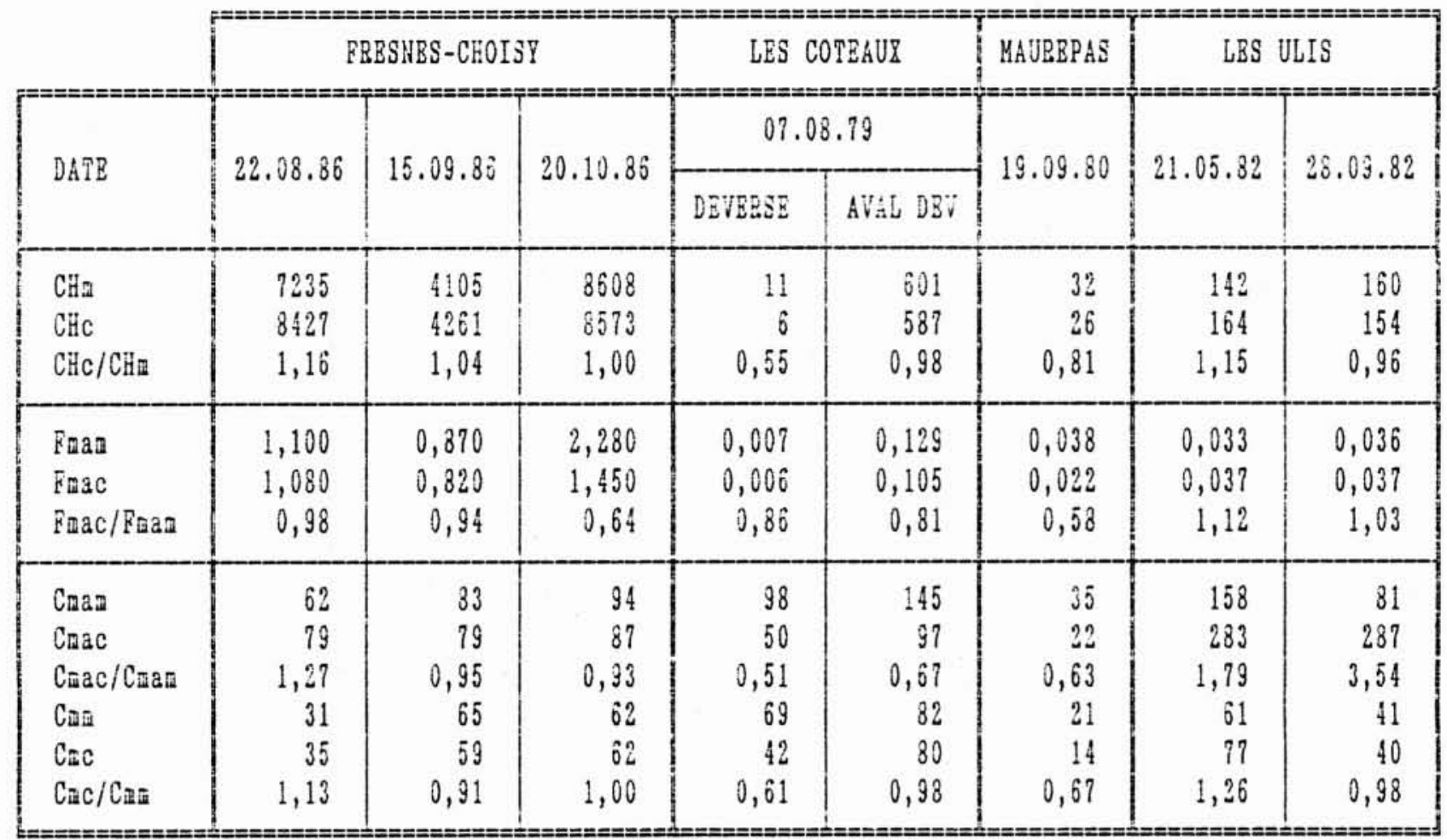


TABLEAU 5 * : DEMANDE CHIMIQUE EN OXYGENE (DCO)

\begin{tabular}{|c|c|c|c|c|c|c|c|c|}
\hline \multirow{3}{*}{$\begin{array}{l}========== \\
D A T B\end{array}$} & \multicolumn{3}{|c|}{ FRESWRS-CHOISP } & \multirow{2}{*}{\multicolumn{2}{|c|}{$\begin{array}{c}\text { LES CUTEAUR } \\
0=============== \\
07.08 .79\end{array}$}} & \multirow{3}{*}{$\begin{array}{l}\text { HAUEEPAS } \\
19.09 .80\end{array}$} & \multicolumn{2}{|c|}{ LES ULIS } \\
\hline & \multirow{2}{*}{22.03 .86} & \multirow{2}{*}{15.09 .80} & \multirow{2}{*}{20.10 .86} & & & & \multirow{2}{*}{21.05 .82} & \multirow{2}{*}{28.09 .82} \\
\hline & & & & DRVEESB & AVAL DBV & & & \\
\hline $\mathrm{CH}$ - & 38424 & 24802 & 55312 & 72 & 4269 & 256 & 854 & 784 \\
\hline & 41565 & 23773 & 49251 & 35 & 2638 & 257 & $125 \hat{0}$ & 1046 \\
\hline $\mathrm{CHC} / \mathrm{CHa}$ & 1,08 & 0,96 & 0,89 & 0,49 & 0,62 & 1,00 & 1,47 & 1,33 \\
\hline Fลa & 5,300 & 5,290 & 13,940 & 0,036 & 0,753 & 0,288 & 0,232 & 0,247 \\
\hline Frac & 5,420 & 4,420 & 9,630 & 0,032 & 0,837 & 0,214 & 0,329 & 0,341 \\
\hline Frac/Fuan & 0,85 & 0,84 & 0,69 & 0,89 & 0,85 & 0,74 & 1,42 & 1,38 \\
\hline Cana & 357 & 505 & 575 & 359 & 910 & 219 & 536 & 547 \\
\hline Conac & 352 & 453 & 450 & 293 & 470 & 212 & $7 \hat{1} 1$ & 754 \\
\hline Crac/Crar & 0,99 & 0,30 & 0,78 & 0,52 & 0,52 & 0,97 & 1,20 & 1,38 \\
\hline Cona & 165 & 394 & 397 & 453 & 585 & 168 & 367 & 202 \\
\hline $\mathrm{Cac}$ & 175 & 328 & 354 & 243 & 359 & 142 & 591 & 270 \\
\hline $\mathrm{Cac} / \mathrm{Cman}$ & 1,06 & 0,83 & 0,83 & 0,54 & 0,61 & 0,85 & 1,61 & 1,34 \\
\hline
\end{tabular}

TABLEAU $6 *$ : AZOTE KJELDHAL (NTK)

\begin{tabular}{|c|c|c|c|}
\hline \multirow{2}{*}{ DATE } & \multicolumn{3}{|c|}{ FRESAES-CHOISY } \\
\hline & 22.08 .86 & 15.09 .86 & 20.10 .85 \\
\hline CHa & 1334 & 746 & 1259 \\
\hline $\mathrm{CHC}$ & 1535 & 754 & 1624 \\
\hline $\mathrm{CH} / \mathrm{CH}$ i & 1,15 & 1,01 & 1,29 \\
\hline Fman & 0,18 & 0,11 & 0,31 \\
\hline Faac & 0,172 & 0,129 & 0,284 \\
\hline Frac/Fman & 0,95 & 1,17 & 0,31 \\
\hline Caan & 10,2 & 15,6 & 12,9 \\
\hline Cmac & 17,1 & 13,0 & 13,1 \\
\hline Crac/Caan & 1,68 & 0,83 & 1,02 \\
\hline Can & 5,7 & 11,9 & 9,0 \\
\hline $\mathrm{Cmc}$ & 6,5 & 10,4 & 11,7 \\
\hline $\mathrm{Cac} / \mathrm{Cmin}$ & 1,14 & 0,87 & 1,30 \\
\hline
\end{tabular}
NOTA : Le bassin du collecteur Fresnes-Choisy est le seul sur lequel des aesures de $N T R$ ont été réalisées.


Theorie des Fleuves

Planike ITI.

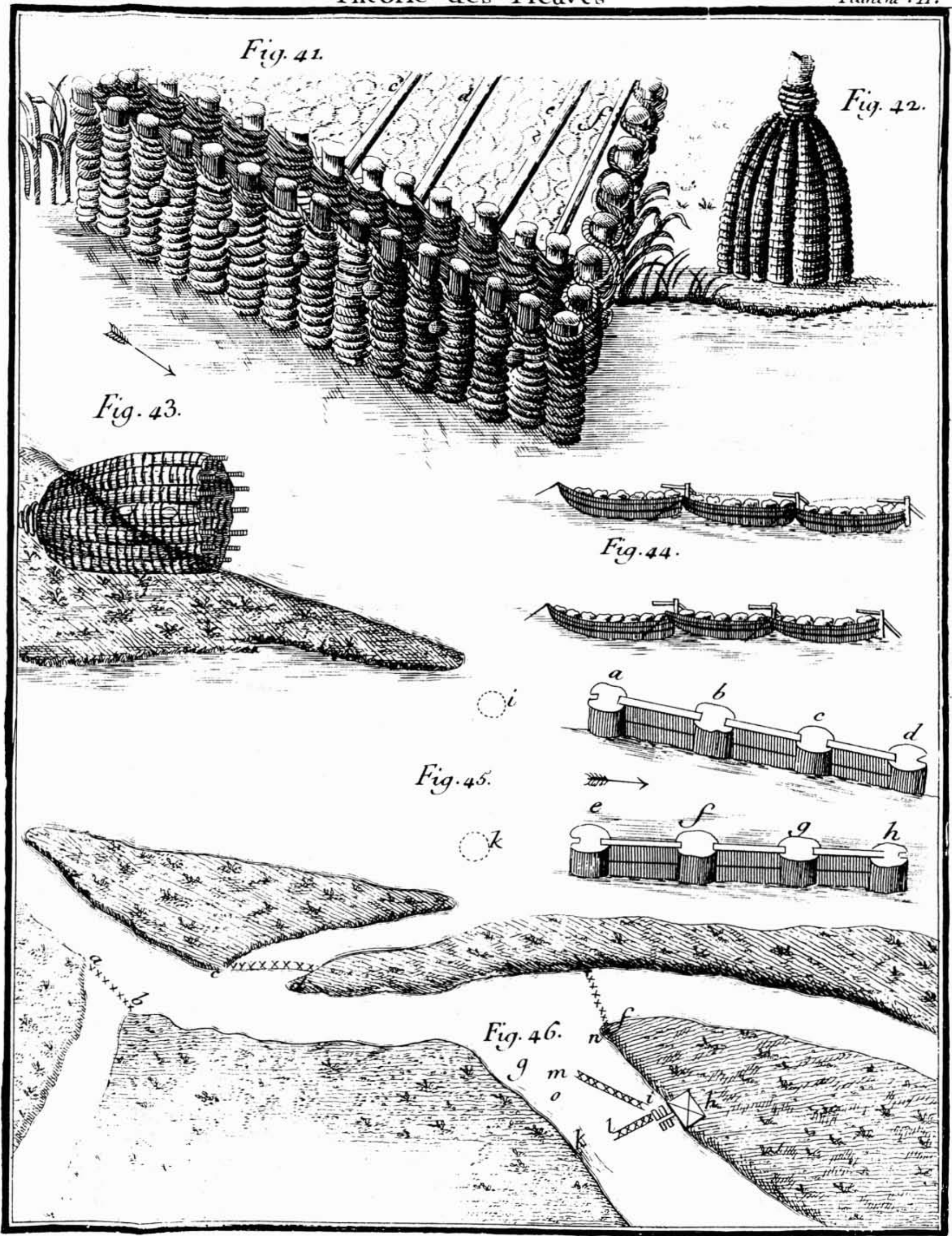

